\title{
Pointwise Regularity Exponents and Well-Behaved Residuals in Stock Markets
}

\author{
Sergio Bianchi and Alexandre Pantanella
}

\begin{abstract}
The article deals with a class of stochastic processes, the Multifractional Processes with Random Exponent (MPRE), recently introduced to gain flexibility in modeling many complex phenomena. We claim that MPRE can capture in a very parsimonious way most of the well known financial stylized facts. In particular, we prove that the process unconditional distributions are fat-tailed and high-peaked and show that, as it occurs for asset returns, the empirical autocorrelation functions of the process increments are close to zero whereas significant values are exhibited by squared (or absolute) increments. Furthermore, we provide evidence that the sole knowledge of functional parameter of the MPRE allows to calculate residuals that perform much better than those obtained by other discrete models such as the GARCH family.
\end{abstract}

Index Terms-Declustering, GARCH, Multifractional Processes with Random Exponents, Residuals

\section{INTRODUCTION}

In a celebrated article, Cont [1] discusses the following eleven stylized facts displayed by financial time series:

1) Absence of (linear) autocorrelations of asset returns, except for small time scales.

2) Heavy tails in the unconditional distribution of returns.

3) Gain/loss asymmetry, meaning that prices are not likely to raise with the intensity they display in falling.

4) Aggregational Gaussianity, meaning that the returns distributions tend to gaussianity as the time scale sampling increases.

5) Intermittency, consisting in the bursts of volatility at any time scale.

6) Volatility clustering, meaning that high-volatility events tend to cluster in time.

7) Conditional heavy tails, consisting in the fact that the residuals obtained correcting the returns for volatility clustering (e.g. via GARCH-type models) still exhibit heavy tailed distributions.

8) Slow decay of autocorrelation in absolute returns, meaning that - contrarily to the behaviour of returns the absolute or squared returns exhibit significant autocorrelation that decays slowly with the lag according to a power law.

Manuscript received January 28, 2011.

Sergio Bianchi is with University of Cassino, Via S. Angelo, 03043 Cassino (Italy) (phone: +39-0776-299-4846; fax: +39-0776-299-4834; e-mail:sbianchi@eco.unicas.it).

Alexandre Pantanella is with University of Cassino, Via S. Angelo, 03043 Cassino (Italy) (phone: +39-07762994845; fax: +39-07762994834; e-mail: a.pantanella@eco.unicas.it).
9) Leverage effect, meaning that most measures of volatility are negatively correlated with the returns.

10) Volume/volatility correlation, according to which the trading volume is correlated with volatility.

11) Asymmetry in time scales, meaning that coarse-grained volatility predict fine-scale volatility better than the other way round.

It is now well known that the Brownian-based standard financial models are ineffective in matching most of the features above recalled. "Anomalies" of returns such as the highly non Gaussian marginal distributions, the absence of autocorrelation and the highly autocorrelated volatility process or the volatility bursts are all well documented in the literature and claim for a rethink of the very bases of standard models.

In order to address the gap between theory and practice, many models have been proposed, by relaxing the assumption of independence or that of identical distribution of the price variations. Just to quote the main research lines of the last quarter of century it suffices to think to the ARCH-like models in discrete time or to the stochastic processes with stable innovations, in continuous time.

In this article we focus on a new class of stochastic processes introduced in 2005 by Ayache and Taqqu [2] without any specific concern to financial time series: the Multifractional Processes with Random Exponent (MPRE). Without entering in details (we will discuss them in Section II), here it suffices to say that the MPRE is a very versatile family of processes able to capture many, if not all, of the stylized facts above recalled.

Roughly speaking, this family of stochastic processes pursues the idea behind the multifractional Brownian motion (mBm [3], [4]) which, in its turn, extended the very well-known fractional Brownian motion ( $\mathrm{fBm}$ ), allowing the Hurst parameter to change in a deterministic way. Basically, the MPRE differs from the $\mathrm{mBm}$ in that it allows the functional Hurst parameter to evolve randomly.

Whereas the $\mathrm{mBm}$ is generally disregarded in finance, mainly because its nonstationarity hampers the inference of global probabilistic properties, the MPRE is decidedly attractive because it has been proved to have stationary increments under a mild technical condition. This is the very first step needed to grasp how to conciliate the no-arbitrage principle with the stochastic kernel used to define the MPRE.

From a practitioner viewpoint, many reasons justify the use of the MPRE as a model of the financial dynamics; detailed discussions can be found in [5], [6], [7], [8] and [9]. Here we restrict ourselves to mention the capability the process has to (a) provide a rationale for the trading 
mechanism, and (b) replicate the stylized facts observed in finance. In this respect, we will prove that the process which can be easily constructed in order to satisfy the stylized facts 3), 4), 5), 6) and 9) - has high-peaked and fat-tailed marginal distributions (stylized fact 2). In addition, we will show that fitting the model on actual financial data also stylized facts 1), 7) and 8) are replicated by the MPRE.

The paper is organized as follows: in Section II, we introduce the model and provide a proof of its distributional properties (high peaks and fat tails); in Section III, we discuss an estimator of the functional parameter of the MPRE and in Section IV we perform an analysis on four main stock indices. Section V concludes.

\section{BACKGROUND ISSUES AND MODEL}

The best way to introduce the Multifractional Process with Random Exponent (MPRE) is perhaps to start from the fractional Brownian motion (fBm), discussed in 1940 by Kolmogorov and revived in 1968 by Mandelbrot and Van Ness [10]. The fBm, denoted in the following by $B_{H}(t)$, is the only Gaussian zero mean, self-similar stochastic process whose covariance function is given by

$$
E\left(B_{H}(t) B_{H}(s)\right)=\frac{K^{2}}{2}\left(|t|^{2 H}+|s|^{2 H}-|t-s|^{2 H}\right)
$$

where $K^{2}=\operatorname{Var}\left(B_{H}(1)\right)$ and $t, s \in \mathbb{R}^{+}$. The moving average representation of the $\mathrm{fBm}$ reads as ${ }^{1}$

$$
B_{H}(t)=K V_{H} \int_{\mathbb{R}}\left(|t-x|^{H-\frac{1}{2}} \mathbf{1}_{]-\infty, t]}(s)-|x|^{H-\frac{1}{2}} \mathbf{1}_{]-\infty, 0]}(s)\right) d W(x)
$$

where $\quad V_{H}=(\Gamma(2 H+1) \sin (\pi H))^{1 / 2} / \Gamma\left(H+\frac{1}{2}\right) \quad$ is a normalizing factor, $\mathbf{1}$ is the indicator function and $W(\cdot)$ denotes the ordinary Brownian motion. The variance of the increment process reads as

$$
E\left(\left\{B_{H}(t)-B_{H}(s)\right\}^{2}\right)=K^{2}|t-s|^{2 H} .
$$

To understand the role played by the parameter $H$, it is useful recalling the notion of (pointwise) Hölder exponent. In general terms, the Hölder exponent of the function $h$ measures the degree of irregularity of its graph. More precisely, the function $h: \mathbb{R} \rightarrow \mathbb{R}$ is called a $(\beta, c)$-Hölder function, with $\beta>0$ and $c>0$, if and only if for all $t_{1}, t_{2}$ such that $t_{1}-t_{2}<1$ it holds $\left|h\left(t_{1}\right)-h\left(t_{2}\right)\right| \leq c\left|t_{1}-t_{2}\right|^{\beta}$. From the definition it follows that the stochastic process $X$ has Hölder exponent $\alpha(t)$ defined by

$$
\begin{gathered}
\alpha\left(t_{0}\right)=\sup \left\{\alpha \in[0,1]: \limsup _{h \rightarrow 0} \frac{X\left(t_{0}+h\right)-X\left(t_{0}\right)}{|h|^{\alpha}}=0\right\} . \\
B_{H}(t)=\int_{\mathbb{R}} \frac{e^{i t \xi}-1}{|\xi|^{H+1 / 2}} d W(\xi)
\end{gathered}
$$

For the $\mathrm{fBm}, \alpha(t)=H$ almost surely at any point $t$. This means that the parameter $H$ is informative not only of the intensity of dependence, but also of the regularity of the process' paths; $H=1 / 2$ reduces to the Brownian motion (panel

1 A different representation of the $\mathrm{fBm}$ can also be given in the frequency domain as b of Figure 1), whereas values lower (larger) than $1 / 2$ (panels $a$ and $c$ of Figure 1) indicate antipersistence (persistence), the stronger the more $H$ deviates from $1 / 2$.
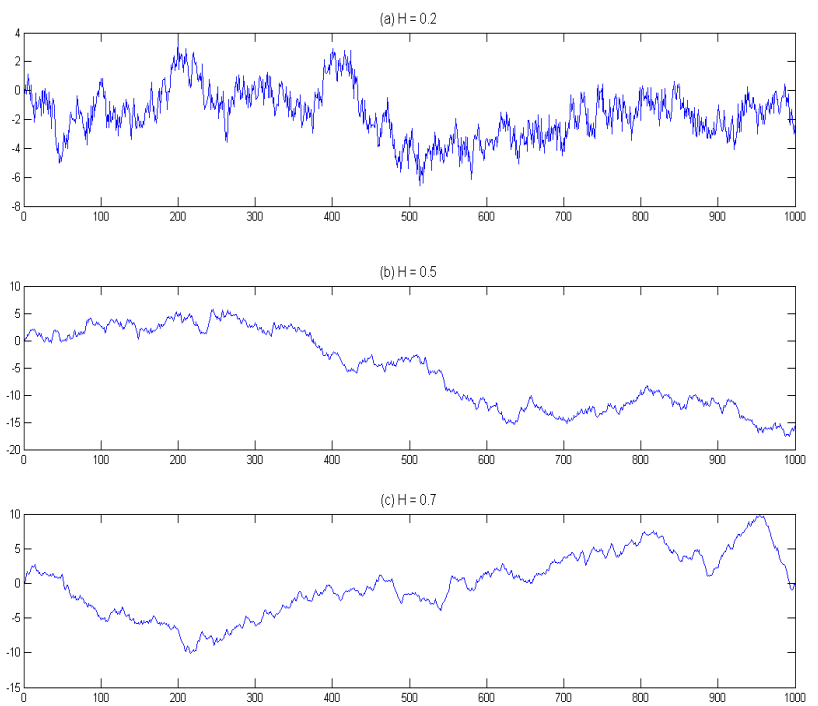

Figure 1. Examples of fBm with different parameters

Since a constant pointwise regularity is too restrictive in the modeling of many phenomena, the $\mathrm{fBm}$ was generalised into the multifractional Brownian motion $(\mathrm{mBm})([3],[4])$, for which the parameter $H$ is replaced by the Hölderian deterministic function $H(t)$. The non anticipative integral representation (1) becomes

$$
\begin{aligned}
& X_{H(t)}(t)=K V_{H(t)} \int_{\mathbb{R}}\left(|t-x|^{H(t)-\frac{1}{2}} \mathbf{1}_{]-\infty, t]}(s)-\right. \\
&\left.-|x|^{H(t)-\frac{1}{2}} \mathbf{1}_{1-\infty, 0]}(s)\right) d W(x)
\end{aligned}
$$

and the pointwise Hölder exponent equals almost surely $H(t)$ at any point $t$. An example of $\mathrm{mBm}$ is given in Figure 2, where the functional parameter has the form $H(t)=1 / 2+$ $0.3 \sin (4 \pi)$. Notice that $H(t)$ tunes the local regularity of the sample path, which in its turn originates the bursts (and the clustering) of variance also in the increment process.

A relevant feature is that the process is asymptotically self-similar with parameter $H(t)$ in a neighborhood of $t$, in the sense stated by [4]. More precisely, denoted by $Y(t, a u)=X_{H(t+a u)}(t+a u)-X_{H(t)}(t)$ the increment process and by $\stackrel{d}{=}$ the equality in distribution, one has

$$
\lim _{a \rightarrow 0^{+}} a^{-H(t)} Y(t+a u) \stackrel{d}{=} B_{H(t)}(u), \quad u \in \mathbb{R}
$$
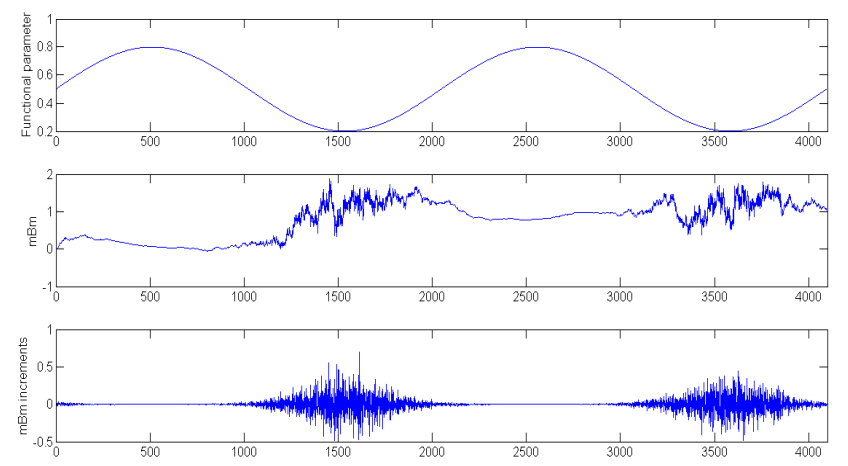

Figure 2. Example of $\mathrm{mBm}$ with sinusoidal functional parameter stating that at any point $t$ an $\mathrm{fBm}$ of parameter $H(t)$ there

where $d W(\xi)$ is a Brownian measure. The harmonizable representation is particularly useful for the analysis of autocorrelation and for simulation purposes. 
exists tangent to the $\mathrm{mBm}$. The result is relevant because it means that all the relations of the $\mathrm{fBm}$ are locally true for the $\mathrm{mBm}$ too. In particular, as a counterpart of relation (2) for the $\mathrm{mBm}$ one has

$$
\mathrm{E}\left(\left\{X_{H(t)}(t)-X_{H(s)}(s)\right\}^{2}\right)=K^{2}|t-s|^{2 H(t)}
$$

for sufficiently small $|t-s|$.

A further step towards flexibility was made in recent years. Exploiting the suggestion by Papanicolaou and Sølna [11] who replace the deterministic function $H(t)$ of the $\mathrm{mBm}$ by a stationary stochastic process with smooth paths and decaying correlation function independent on $W$ - Ayache and Taqqu [2] introduce the Multifractional Processes with Random Exponent (MPRE). Their construction considers (a) the Gaussian field $\left\{B_{\mathcal{H}}(t)\right\}_{(t, \mathcal{H}) \in[0,1] \times[\mathrm{a}, \mathrm{b}] \subset(0,1)}$ depending on $t$ and $\mathcal{H}$, with integral representation provided by (1) and (b) the stochastic process $\{\mathcal{H}(t)\}_{t \in[0,1]}$ with values in the arbitrary fixed compact interval $[a, b]$. Equipped with this notation, the MPRE of parameter $\{S(t)\}_{t \in[0,1]}$ is defined as

$$
Z(t, \omega)=f_{2}\left(f_{1}(t)\right)=B_{\mathcal{H}(t, \omega)}(t, \omega) .
$$

Any trajectory of $Z(t, \omega)$ is the composition of two functions:

$f_{1}:[0,1] \rightarrow[0,1] \times[a, b], t \rightarrow(t, \mathcal{H}(t, \omega))$, that builds the random process which serves as functional parameter, and

$f_{2}:[0,1] \times[a, b] \rightarrow \mathbb{R},(t, \mathcal{H}) \rightarrow B_{\mathcal{H}}(t, \omega)$, that rules the resulting process.

The functional parameter $\mathcal{H}(t, \omega)$ is not necessarily stationary nor independent from the white noise $W$ in (3); if independence is assumed, a particularly relevant result is that the increments of the MPRE form a stationary sequence. This means that - opposite to the case of $\mathrm{mBm}$ - the MPRE can be globally characterized in probabilistic terms. No need to say this is crucial for tackling many problems (first of all, for answering the question whether the process admits arbitrage).
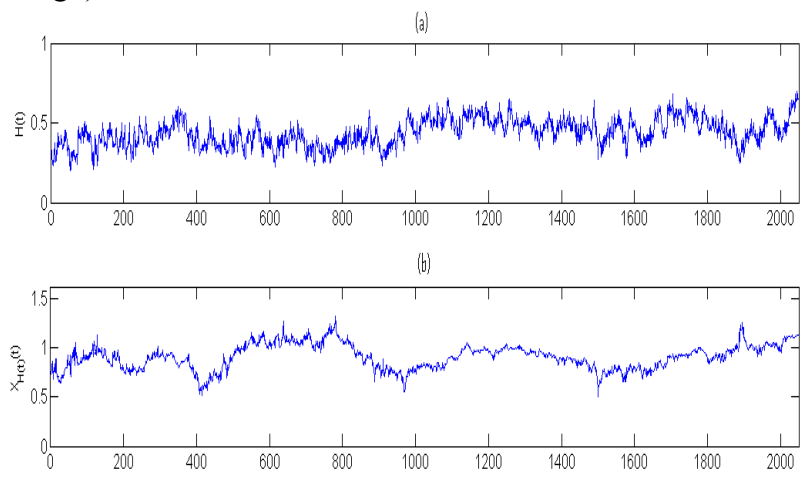

(c)

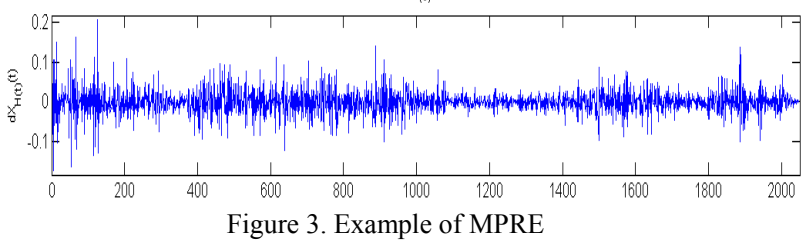

Also the continuity of the MPRE ultimately relies on the continuity of the process that provides its random exponent.

Two issues are relevant for understanding the descriptive power of the MPRE. By construction: (a) the process is able to capture at the same time a very irregular local behavior and long range dependence; (b) relation (6) indicates that the
MPRE behaves locally as an $\mathrm{fBm}$.

This is quite evident from Figure 3, which displays a sample path of MPRE: the random functional parameter $\mathcal{H}(t)$ is shown in panel (a), panel (b) displays the trajectory of the MPRE, and panel (c) reproduces the process increments.

Since it is fundamental to obtain well-behaved trajectories (i.e. trajectories that satisfy the stylized facts), a few words deserve to be spent on the choice of the random functional parameter. Leaded by a number of empirical estimates, as a first approximation (in Figure 3 and in the following) we simulated $\mathcal{H}(t, \omega)$ through properly rescaled samples of fBm of given parameter $H$. More precisely, denoted by $H_{\min }$ and $H_{\text {max }}$ respectively the minimum and the maximum $\mathcal{H}(t, \omega)$ to be surrogated, the procedure works as follows:

- a $B_{H}(t)$ with $H<1 / 2$ is simulated (both the built-in MatLab Wavelet Toolbox ${ }^{\mathrm{TM}}$ and the Chan and Wood algorithm [12] were used in simulations without significant differences);

- in order to force the simulated $\mathcal{H}(t, \bar{\omega})$ to belong to the interval $\left[H_{\min }, \mathrm{H}_{\max }\right]$, the path is rescaled setting

$$
\mathcal{H}(t, \bar{\omega})=\frac{B_{H}(t)-\min _{t} B_{H}(t)}{\max _{t} B_{H}(t)-\min _{t} B_{H}(t)}\left(H_{\text {max }}-H_{\text {min }}\right)+H_{\text {min }}
$$

- for each $t$, the MPRE is simulated as $B_{\mathscr{H}(t, \bar{\omega})}(t, \omega)$.

Notice that, for both $\mathrm{mBm}$ and MPRE, while the bursts of variance in the increments are induced by values of $\mathcal{H}(t, \omega)$ smaller than $1 / 2$, the clustering of variance is induced by the continuity of the process by which $\mathcal{H}(t, \omega)$ is simulated. In fact, the continuity of $\mathcal{H}(t, \omega)$ ensures that values close one each other share approximately the same regularity. As a consequence of the time-changing regularity, high peaks and heavy tails appear in the unconditional distribution (Figure 4 and the proof that follows).

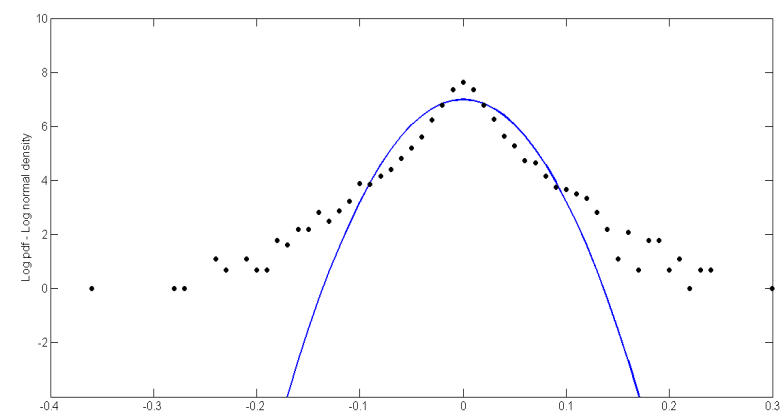

Figure 4. Log-pdf of the MPRE (dots) increments compared with the normal distribution (line)
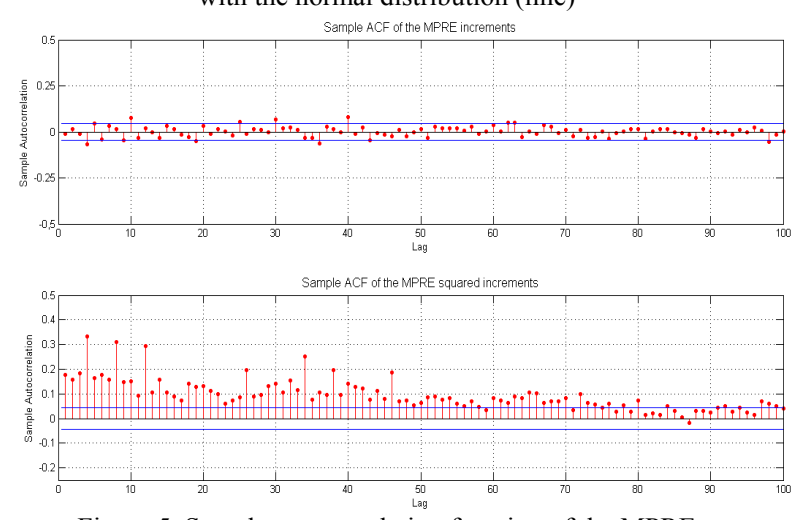

Figure 5. Sample autocorrelation function of the MPRE

We also notice, but the result has not been proved yet (at least in authors' knowledge), that the sample autocorrelation function of the surrogated series (Figure 5) is characterized 
by the absence of linear dependence in its increments but by significant dependence in its squared increments (this continues to be empirically true even when one replaces the squared increments by the absolute ones).

Since at each time $t, \mathcal{H}(t)$ summarizes the process pointwise regularity, this measure can be interpreted as the weight that investors assign to past prices in taking their trading decisions. When $\mathcal{H}(t)$ is larger than $1 / 2$, traders are confident that future information will confirm past positions and the market, which generally underreacts in these circumstances, originates trends and persistence; when $\mathcal{H}(t)$ equals $1 / 2$ all past information is fully reflected in the price, which is not useful to predict future trends (the market is efficient and follows a Brownian motion). Finally, when $\mathcal{H}(t)$ is smaller than $1 / 2$, traders expect that future information will contradict past positions and for this reason their attitude is to correct previous positions by acting oppositely. This is the very mechanism that generates overreaction and mean reversion due to the quick buy-and-sell strategy (the so called touch-and-go market) carried out by investors (see [13]).

A time-changing Hölder exponent produces unconditional distributions with high peaks and fat tails. To prove this, one can exploit property (4) and consider the queueing of $n$ fractional Brownian motions having different parameters, i.e. different Hölder exponents. Sampling the process at $n>1$ points on the unit interval so that $t_{i}=i / n(i=0, \ldots, n)$, set $H(t)$ as piecewise constant

$$
H\left(t_{i}\right)=\sum_{i=1}^{n} H_{i} \mathbf{1}_{\left[t_{i-1}, t_{i}\right)}^{(t)} .
$$

Recalling relation (2), the variance of the unconditional distribution trivially reads as

$$
\sigma^{2}=K^{2} \sum_{i=1}^{n}\left(t_{i}-t_{i-1}\right)(n-1)^{-2 H_{i}}=\sum_{i=1}^{n}\left(t_{i}-t_{i-1}\right) \sigma_{i}^{2}
$$

where $\sigma_{i}^{2}=K^{2}(n-1)^{-2 H_{i}}$. Finally, the unconditional density can be written as

$$
f_{X}(x)=\sum_{i=1}^{n} \frac{\left(t_{i}-t_{i-1}\right)}{\sigma_{i} \sqrt{2 \pi}} e^{-\frac{x^{2}}{2 \sigma_{i}^{2}}} .
$$

(Remind that we are not considering the combination of the sequences but just their queueing).

Step 1 (leptokurtosis)

Let us prove that $f_{X}(x)$ is leptokurtic with respect to the Gaussian distribution. To this aim consider the index of kurtosis excess $\gamma_{2}=\mathrm{E} X^{4} / \sigma^{4}-3$. Values of $\gamma_{2}$ larger than zero denote that the kurtosis is larger that the one of the Gaussian distribution. Let us calculate $\mathrm{E} X^{4}$ by the moment-generating function of (6):

$$
\begin{aligned}
m_{X}(t) & =\mathrm{E}\left(e^{t X}\right)=\int_{\mathbb{R}} e^{t x} f_{X}(x) d x \\
& =\int_{\mathbb{R}} e^{t x} \sum_{i=1}^{n} \frac{\left(t_{i}-t_{i-1}\right)}{\sigma_{\mathrm{i}} \sqrt{2 \pi}} e^{-\frac{x^{2}}{2 \sigma_{i}^{2}}} d x \\
& =\int_{\mathbb{R}} \sum_{i=1}^{n} \frac{\left(t_{i}-t_{i-1}\right)}{\sigma_{\mathrm{i}} \sqrt{2 \pi}} e^{-\frac{\left(x^{2}-\sigma_{i}^{2}\right)^{2}}{2 \sigma_{i}^{2}}+\frac{\sigma_{i}^{2} t^{2}}{2}} d x \\
& =\sum_{i=1}^{n}\left(t_{i}-t_{i-1}\right) e^{\frac{\sigma_{i}^{2} t^{2}}{2}} \int_{\mathbb{R}} \frac{1}{\sigma_{i} \sqrt{2 \pi}} e^{-\frac{\left(x^{2}-\sigma_{i}^{2}\right)^{2}}{2 \sigma_{i}^{2}}} d x
\end{aligned}
$$

As the integrands in the last line are normal densities with mean $\mu_{i}=\sigma_{i}^{2} t$, the integrals equals one. It follows that

$$
m_{X}(t)=\mathrm{E}\left(e^{t X}\right)=\sum_{i=1}^{n}\left(t_{i}-t_{i-1}\right) e^{\frac{\sigma_{i t}^{2} t^{2}}{2}} .
$$

Recalling that $\frac{d^{k} m}{d t^{k}}(0)=\mathrm{E}\left(X^{k}\right)$, one has

$$
\frac{d^{4} m}{d t^{4}}(0)=\mathrm{E}\left(X^{4}\right)=\sum_{i=1}^{n}\left(t_{i}-t_{i-1}\right) 3 \sigma_{i}^{4}
$$

Substituting in the index $\gamma_{2}$ and solving the inequality

leads to

$$
\frac{3 \sum_{i=1}^{n}\left(t_{i}-t_{i-1}\right) \sigma_{i}^{4}}{\left(\sum_{i=1}^{n}\left(t_{i}-t_{i-1}\right) \sigma_{i}^{2}\right)^{2}}-3>0
$$

$$
\sum_{i=1}^{n}\left(t_{i}-t_{i-1}\right) \sigma_{i}^{4}>\left(\sum_{i=1}^{n}\left(t_{i}-t_{i-1}\right) \sigma_{i}^{2}\right)^{2} .
$$

As by virtue of the Cauchy inequality

$$
\left(\sum_{i=1}^{n}\left(t_{i}-t_{i-1}\right) \sigma_{i}^{2}\right)^{2} \leq \sum_{i=1}^{n}\left(t_{i}-t_{i-1}\right)^{2} \sum_{i=1}^{n} \sigma_{i}^{4},
$$

it suffices to prove

$$
\sum_{i=1}^{n}\left(t_{i}-t_{i-1}\right) \sigma_{i}^{4}>\sum_{i=1}^{n}\left(t_{i}-t_{i-1}\right)^{2} \sum_{i=1}^{n} \sigma_{i}^{4},
$$

which trivially holds, since from $t_{i}-t_{i-1}=\frac{1}{n}$ it follows

$$
\sum_{i=1}^{n} \sigma_{i}^{4}>\frac{1}{n} \sum_{i=1}^{n} \sigma_{i}^{4}
$$

Since the index of kurtosis is larger than zero, the unconditional distribution is leptokurtic with respect to the normal law.

Step 2 (fat tails)

In order to prove that the unconditional distribution exhibits also fat tails with respect to the Gaussian density, it suffices to define the ratio

$$
Q(x)=\frac{\sum_{i=1}^{n} \frac{t_{i}-t_{i-1}}{\sigma_{i} \sqrt{2 \pi}} e^{-\frac{x^{2}}{2 \sigma_{i}^{2}}}}{\frac{1}{\sigma \sqrt{2 \pi}} e^{-\frac{x^{2}}{2 \sigma^{2}}}}
$$

Since $\sigma^{2}$ is the convex combination of the values $\sigma_{i}^{2}$, properly arranging these one can write

from which it follows

$$
\sigma_{1}<\ldots<\sigma_{k}<\sigma<\sigma_{k+1}<\ldots<\sigma_{n}
$$

$$
Q(x)=\sigma\left(\sum_{i=1}^{k} \frac{\left(t_{i}-t_{i-1}\right) e^{-\frac{\left(\sigma_{i}^{2}-\sigma^{2}\right)^{2}}{2 \sigma_{i}^{2} \sigma^{2}}}}{\sigma_{i}}+\sum_{i=k+1}^{n} \frac{\left(t_{i}-t_{i-1}\right) e^{-\frac{\left(\sigma_{i}^{2}-\sigma^{2}\right) x^{2}}{2 \sigma_{i}^{2} \sigma^{2}}}}{\sigma_{i}}\right)
$$

and therefore

$$
\lim _{x \rightarrow \infty} Q(x)=+\infty
$$

Limit (7) states that the function at the numerator of $Q(x)$ (i.e. the density obtained by queueing the sequences) tends to zero more slowly than the function at the denominator (i.e. the normal distribution) as $x$ diverges, and this trivially indicates that the tails are heavier, namely the model satisfies stylized fact 2). 


\section{Estimation OF $\mathcal{H}(t, \omega)$}

By arguments discussed in the previous section, it come quite evident that the knowledge of the random functional parameter is essential in order to conveniently employ MPRE in financial modeling. As noted, the functional parameter $\mathcal{H}(t, \omega)$ provides a measure of the process pointwise regularity and this is the very rationale for the financial interpretation given above. Assuming the constancy of $\mathcal{H}(t, \omega)$ with respect to time, that is assuming the price process to be an $\mathrm{fBm}$, many contributions in past years tried to estimate the overall regularity of financial time series, but a number of inconsistent results were obtained (see, e.g. [5, 14] for a survey). In particular, taking into account twenty-three works, [5] conclude that the estimates are considerably different and range in the interval $[0.41,0.70]$ (remind that $H$ belongs to the interval $[0,1])$. Other analyses $([5,6,15,16])$ show that the functional parameter fluctuates around $1 / 2$, rarely overstays for long periods above this threshold and displays on the contrary large downward movements when market crashes occur. This behavior seems to be strongly consistent with how actual financial markets do operate. In fact, if confidence in the past is a gradually increasing process, the destroy of memory is likely to be a sudden event, often caused by shocking innovations (such as, for example, a destabilizing political news or the release of negative financial reports).

With these premises in mind, the estimation of $\mathcal{H}(t, \omega)$ plays a fundamental role in the tangible use of the MPRE. Roughly speaking, it is about estimating the trajectory of panel (a) of Figure 3 by knowing the sample path of the process (panel (b)). In order to accomplish this step we can use the estimator introduced in [5] and recently improved in [16], contributions to which we refer for a detailed description of the estimator. Here, we will just recall that given the time series $\left\{X_{j, n}\right\}$ with $n$ data sampled in discrete time and with unit time variance equal to $K^{2}-$ the sliding-window estimator reads as

$$
H_{\delta, q, n, K}^{k}(t)=\frac{\log \left(\frac{\sqrt{\pi}}{\delta-q+1} \sum_{j=t-\delta}^{t-1}\left|X_{j+q, n}-X_{j, n}\right|^{k} / 2^{k / 2} \Gamma\left(\frac{k+1}{2}\right) K^{k}\right)}{k \log \left(\frac{q}{n-1}\right)}
$$

where $q$ is the order of the lagged increments and $\delta$ is the length of the estimation window.

Estimator defined by (8): assumes the increments process $X_{j+q, n}-X_{j, n}$ to be normally distributed within the window $\delta$ with mean zero and
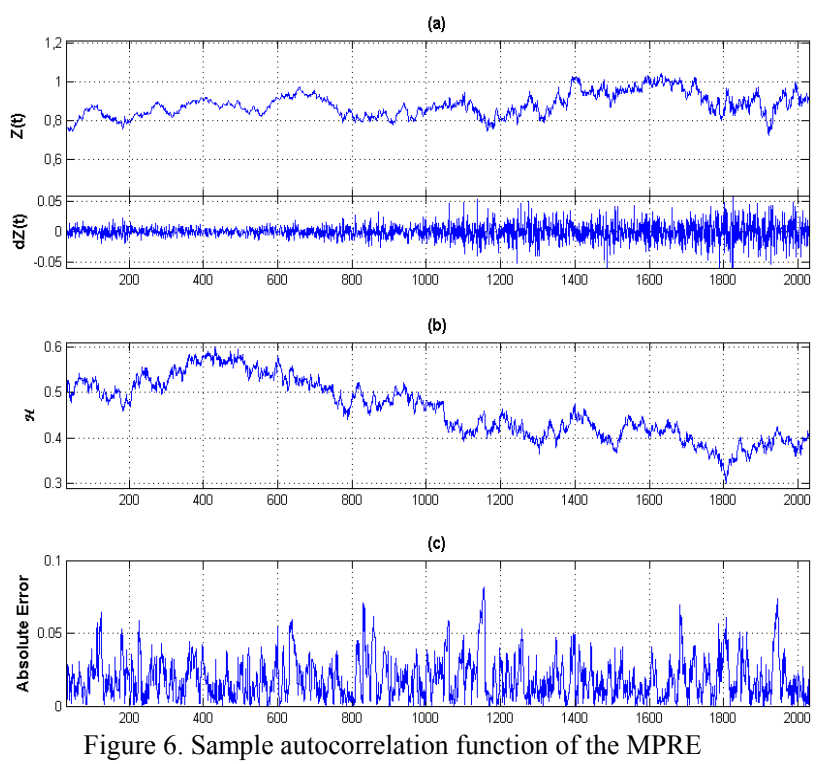

variance given by relation (5). Notice that this is consistent with the assumption that locally, that is in a neighborhood of each sampled point $t$, the process $X$ behaves as an $\mathrm{fBm}$; is normally distributed with mean equal to the parameter to be estimated and variance that when $H(t)=1 / 2$ equals to

$$
\operatorname{Var}\left(H_{\delta, 1, n, 1}^{k}(t)\right)=\frac{\sqrt{\pi}}{\delta k^{2} \log ^{2}(n-1) \Gamma^{2}\left(\frac{k+1}{2}\right)}\left[\Gamma\left(\frac{2 k+1}{2}\right)-\frac{1}{\sqrt{\pi}} \Gamma^{2}\left(\frac{k+1}{2}\right)\right]
$$

For the other cases, confidence intervals depending on $n, \delta$ and $H(t)$ are provided by Monte Carlo simulations in [16]; has a $(\sqrt{\delta} \log n)$-rate of convergence, $\delta$ and $n$ respectively being the length of the estimation window and the number of sampling points.

An idea of how estimator (8) works is provided in Figure 6: panel (a) displays a trajectory of $Z(t, \omega)$ with its increments; panel (b) shows the functional parameter $\mathcal{H}(t, \bar{\omega})$ and panel (c) the absolute error of the estimates provided by $H_{20,1,2048,0.5}^{2}(t)$. The overall mean absolute error is $2.14 \times 10^{-2}$.

Once the estimation of $\mathcal{H}(t, \omega)$ was obtained through (8), we used it to calculate the residuals in the usual way

$$
r_{t}=\frac{d X_{t, n}}{K(n-1)^{-H_{\delta, q, n, K}^{k}(t)}} .
$$

If the model and the estimator work, we obviously expect $r_{t}$ to be i.i.d. standard normal. We checked this result using the Jarque-Bera normality test [17] (identical distribution) and evaluating the sample autocorrelation functions of both the residuals and squared residuals (independence).

The Jarque-Bera test quantifies the distance from the Gaussian distribution in terms of skewness and kurtosis and then computes a single $p$ value using the sum of the discrepancies.

Analyzing autocorrelation functions serves a different purpose. It is well-known that heavy tails and volatility clustering, the very motivations for the use for example of GARCH models, are expected to disappear once the returns are normalized by the level of volatility, making their distribution Gaussian. Nevertheless, under the classical ARMA-GARCH modeling, such scaled returns are still generally heavy tailed and show extremal dependence, whose strength reduces only as extreme levels increase. This unpleasant result, which constitutes a serious warning about the capability of such models to capture entirely the variation 
in volatility, is generally addressed by modeling the residuals by some ad hoc distribution. The most popular ones used to this aim are the (eventually asymmetric) Student's $t[18,19$, $20,21]$, the generalized Pareto [22, 23], the normal inverse Gaussian distribution [24] or the double exponential, as a particular case of the generalized error distribution when the tail thickness parameter equals 1 [25]. In spite of the many efforts devoted to the analysis of this topic, nevertheless the choice of such distributions appears quite arbitrary and - in authors' opinion - makes questionable the capability of the above recalled models to capture the real nature of the volatility dynamics.

\begin{tabular}{lcccccc} 
& \multicolumn{7}{c}{ DABLE I. DATA } \\
\cline { 2 - 7 } & Obs. & Mean & St. Dev & Kurt & Skew & $\begin{array}{c}\text { Tail } \\
\text { index }\end{array}$ \\
\hline DJIA & 1,379 & 0.0000 & 0.0137 & 12.802 & 0.0246 & 0.2252 \\
\hline FTSE & 1,384 & 0.0000 & 0.0139 & 11.117 & -0.1084 & 0.2417 \\
\hline N225 & 1,342 & -0.0001 & 0.0172 & 11.362 & -0.4255 & 0.2161 \\
\hline HSI & 1,374 & 0.0003 & 0.0187 & 11.210 & 0.0838 & 0.2507 \\
\hline
\end{tabular}

\section{EMPIRICAL APPLICATION}

With regard to the last five years (01/02/2005$06 / 30 / 2010$ ), we analyzed the four main financial stock indices, whose distributional parameters are summarized in Table 1. No discussion about the highly non Gaussian behavior of the log-increments for all the series.

We estimated the pointwise regularity of each index, setting $\delta=15$ and $q=1$ (the right $K$ was estimated using the technique proposed in [16]). The estimates of $H_{\delta, q, n, K}^{k}(t)$ are displayed in Figure 7.
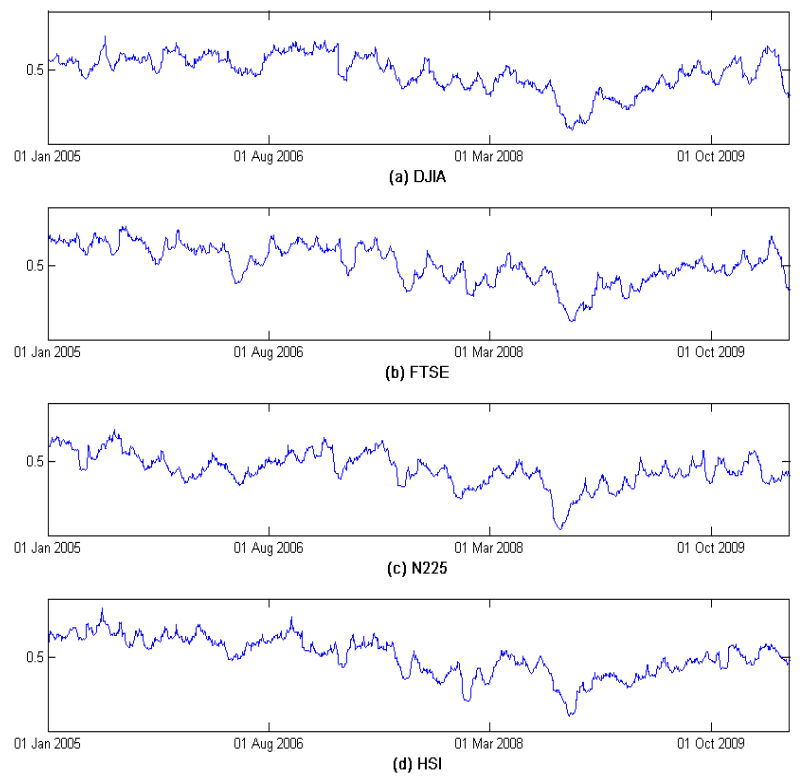

Figure 7. Pointwise regularity estimates of the four indices

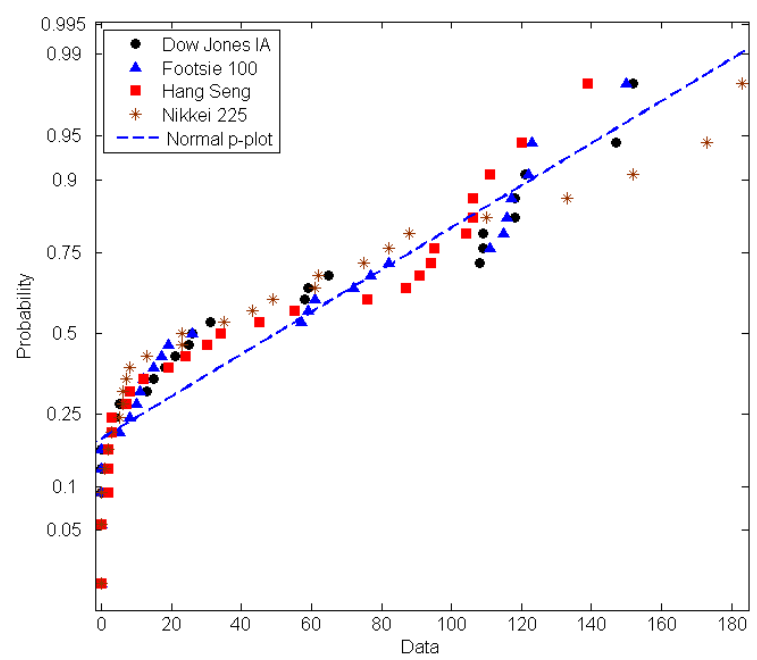

Figure 8. Probability plot of the estimated $\mathcal{H}(t)$

\begin{tabular}{|c|c|c|c|c|}
\multicolumn{1}{c|}{ TABLE II. CORRELATION MATRIX } \\
\cline { 2 - 5 } \multicolumn{1}{c|}{} & DJI & FTSE & N225 & HSI \\
\hline DJI & 1,000 & $\begin{array}{c}0.903 \\
(0.401)\end{array}$ & $\begin{array}{c}0.834 \\
(0.395)\end{array}$ & $\begin{array}{c}0.792 \\
(0.227)\end{array}$ \\
\hline FTSE & - & 1,000 & $\begin{array}{c}0.891 \\
(0.362)\end{array}$ & $\begin{array}{c}0.835 \\
(0.442)\end{array}$ \\
\hline N225 & - & - & 1,000 & $\begin{array}{c}0.795 \\
(0.516)\end{array}$ \\
\hline HSI & - & - & - & 1,000 \\
\hline
\end{tabular}

Notice that in all the cases the pointwise regularity fluctuates around $1 / 2$, the value for which the MPRE reduces to the Brownian motion and all work in terms of absence of arbitrage [26]. The sudden drops in the parameter, observed in correspondence of financial crashes, are followed by more gradual upward movements, consistently with the financial interpretation discussed in Section II. The asymmetric behavior of the functional parameter trajectories reflects itself in Figure 8, which displays the probability plots of the estimates compared with the normal distribution. While differences appear in the center and in the right-hand side of the distributions, the left tails behave almost in the same way for all the indices, what indicates homogeneity of reaction when shocks occur.

Also consider Table II, that displays the values of the correlation matrix in terms of both estimated functional parameter and volatility (in parentheses). The fact that the former values are decidedly higher than the latter is symptomatic of the insight provided by $\mathcal{H}(t)$ estimates; they do not merely account for the volatility level of the market, but give information about how investors perceive incoming news with respect to past positions. In fact, the latent variable $\mathcal{H}(t)$ seizes how different markets perceive (one with respect to each other) the past information conditional to the arrival of new information. This inner knowledge permits to evaluate how accordingly markets discount the past [15]. 


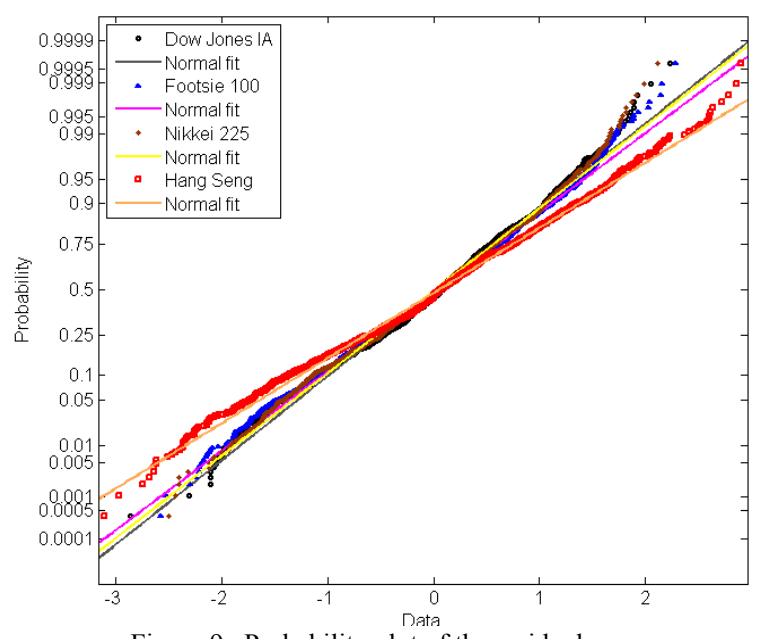

Figure 9. Probability plot of the residuals

The second step of the empirical analysis consisted in calculating the residuals (9). Table III summarizes their distributional properties, also displayed in Figure 8 in terms of probability plot. Due to the nonlinear transformation induced by the estimator (8) on the log-variations, the mean differs from the original value but is still close to zero. While the skewness generally differs from zero (even if, as it will be seen in a while, the difference is not significant), notice that the standard deviation is approximately one and the kurtosis is very close to 3 , values of the $N(0,1)$ law.

In order to check for the normality of the residuals we performed the Jarque-Bera test for increasing time spans (from 20 trading days to about four trading years, i.e. 1,000 observations). The results, summarized in Tables IV, show that - except for the Dow Jones, which is normally distributed at $\alpha=0.05$ for about one trading year - all the other indices have residuals which are standard normal for the overall period. The results are compared with those obtained fitting the time series by GARCH(1,1) (Table V). Notice that for almost all the sizes the MPRE residuals perform better than the corresponding GARCH residuals.

The last analysis concerned the autocorrelation of both the residuals and the squared residuals. Figures 10 and 11 display the results: in all the cases the residuals are not autocorrelated (we used the of Box, Jenkins, and Reinsel [27] estimator). This supports the conclusion that - as expected - they are i.i.d.
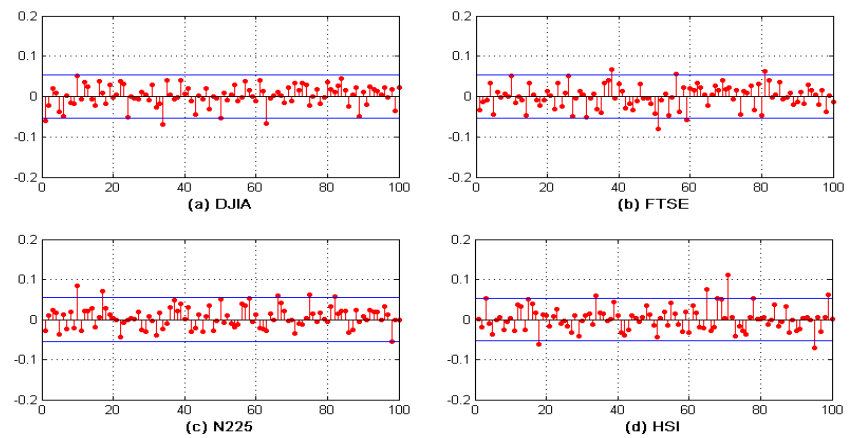

Figure 10. Sample autocorrelation functions of the residuals
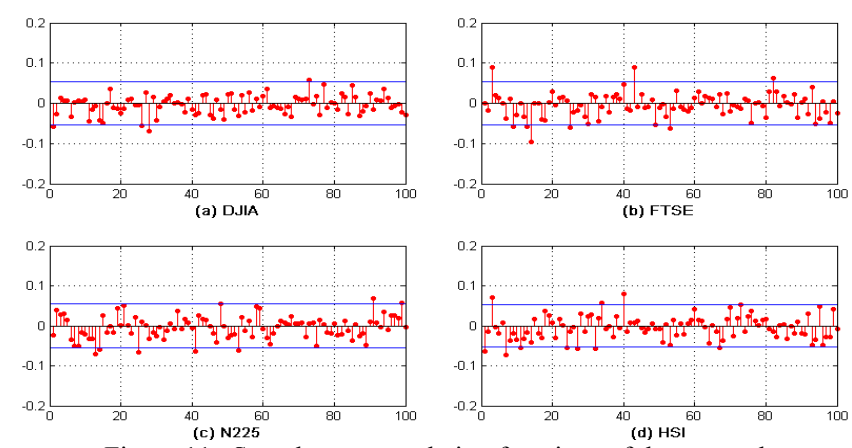

Figure 11. Sample autocorrelation functions of the squared Residuals

\section{CONCLUSION AND Further DEVELOPMENTS}

In this paper we have proposed to model the financial time series using a very versatile stochastic process, the MPRE, recently introduced by [2]. In author's knowledge it is the very first time that this process is used in finance and our preliminary findings, in terms of well-behaved distributions as well as the goodness of fit of the sample autocorrelation functions (of the increments and of the squared ones), indicate that the model really deserves more attention. After discussing the main properties of the model and proving that the variability of its pointwise regularity exponent produces non Gaussian unconditional distributions, we used a robust estimator of the MPRE functional parameter in order to quantify the pointwise regularity of four main stock indices. We found that the estimated parameters consistently describe the market behavior (we provide a financial intuition of this) and synthesize the volatility process. The pointwise regularity also serves to analyze the residuals of the historical data. Since we found evidence that they are i.i.d. standard normal until a time horizon of about one trading year in the worst case of DJIA, of four trading years for the FTSE and for the whole sample for the N225 and the HSI, we conclude that the model succeeds in capturing locally the volatility process better than GARCH-like models. The empirical evidence suggests that, when the MPRE is assumed as a model, the autocorrelation functions of $\log$ price variations and variance behave accordingly to stylized facts well-known in finance. Further work is necessary to set these empirical findings in a theoretical framework.

\section{REFERENCES}

[1] R. Cont, "Empirical properties of asset returns: stylized facts and statistical issues". Quantitative Finance, 1(2), 2001, pp.223-236

[2] A. Ayache, M. Taqqu, "Multifractional processes with random exponent", Publicacions Matemàtiques, 49, 2005, pp.459-486.

[3] R. Péltier, J. Lévy Véhel, "Multifractional brownian motion: definition and preliminary results", Technical Report RR-2645, INRIA, 1995.

[4] A. Benassi, S. Jaffard, and D. Roux, "Elliptic gaussian random processes”, Rev. Mat. Iberoamericana, 13, 1997, pp.19-90.

[5] S. Bianchi, "Pathwise identification of the memory function of multifractional brownian motion with application to finance", International Journal of Theoretical and Applied Finance (IJTAF), 8(2), 2005, pp.255-281.

[6] S. Bianchi and A. Pianese, "Multifractional properties of stock indices decomposed by filtering their pointwise Hölder regularity", International Journal of Theoretical and Applied Finance (IJTAF), 11(6), 2008, pp.567-595. 
[7] R.L. Costa and G. L. Vasconcelos, "Long-range correlations and nonstationarity in the brazilian stock market", Physica A: Statistical Mechanics and its Applications, 329(1-2), 2003, pp.231-248.

[8] A.F. Crepaldi, C.R. Neto, F.F. Ferreira and G. Francisco, "Multifractal regime transition in a modified minority game model", Chaos, Solitons \& Fractals, 42(3), 2009, pp.1364-1371

[9] A. Pantanella and A. Pianese, "Minimum risk portfolios using MMAR", Proceedings of the 10th WSEAS international conference on Mathematics and computers in business and economics, World Scientific and Engineering Academy and Society (WSEAS), 2009, pp.64-71

[10] B. Mandelbrot and J. Van Ness, "Fractional brownian motions, fractional noises and applications", SIAM Review, 10(4), 1968, pp.422-437.

[11] G.C. Papanicolaou and K. Sølna, "Wavelet based estimation of local Kolmogorov turbulence", in Theory and applications of long-range dependence, Birkhäuser Boston, Boston, MA, 2003, pp. 473-505.

[12] G. Chan and A. Wood, "Simulation of multifractional brownian motion", in Proceedings in Computational Statistics, (1998), 233-238

[13] S. Bianchi and A. Pantanella, "Efficiency, Overreaction and Underreaction in Stock Markets. A Parsimonious Model of the Three Sided-Coin", forthcoming in ICFTE 2011, Shanghai (China), 11-13 May 2011

[14] M. Henry and P. Zaffaroni, "The long range dependence paradigm for macroeconomics and finance", in Long range dependence: Theory and applications, P. Doukhan, G. Oppenheim and M. Taqqu (ed). Birkhäuser, 2002, Boston

[15] A. Pantanella and S. Bianchi, "Pointwise Regularity Exponents and Markets Cross-Correlation", International Review of Business Research Papers, vol. 6(2), 2010, 39-51

[16] S. Bianchi, A. Pantanella and A. Pianese, "Modeling stock prices by multifractional Brownian motion: an improved estimation of the pointwise regularity", submitted

[17] C.M. Jarque and A.K. Bera, "A test for normality of observations and regression residuals", International Statistical Review 55, 1987, $163-172$

[18] T. Bollerslev, A Conditionally Heteroskedastic Time Series Model For Speculative Prices and Rates of Return, Review of Economics and Statistics 69, 1987, 542-547

[19] T. Bollerslev, R. Chou and K. Kroner, ARCH modelling in finance: a review of theory and empirical evidence, Journal of Econometrics, 52, 1992, 5-60

[20] P. Hall and Q. Yao, Inference in ARCH and GARCH models with heavy-tailed errors, Econometrica, 71, 2003, 285-317

[21] C. Fernandez and M. Steel, On Bayesian Modeling of Fat Tails and Skewness, Journal of the American Statistical Association, 93, 1998, 359-371

[22] F. Laurini and J.A. Tawn, "Regular Variation and Extremal Dependence of GARCH Residuals with Application to Market Risk Measures", Econometric Reviews, 28(1-3), 2009 , 146-169

[23] McNeil, A.J., and R. Frey, (2000), Estimation of tail-related risk measures for heteroscedastic financial time series: an extreme value approach. Journal of Empirical Finance, 7, 271-300

[24] J.H. Venter and P.J. de Jongh, Risk Estimation Using the Normal Inverse Gaussian Distribution, Journal of Risk, 4, (2002), 1-23

[25] D.B. Nelson, "Conditional Heteroskedasticity in Asset Returns: a New Approach", Econometrica, 59(2), 1991, 347-370

[26] L.C.G. Rogers, "Arbitrage with fractional Brownian motion", Mathematical Finance, 7(1), 1997, 95-105

[27] G.E.P. Box, G. M. Jenkins, and G. C. Reinsel, "Time Series Analysis: Forecasting and Control", 3rd ed. Upper Saddle River, NJ: Prentice-Hall, 1994

Sergio Bianchi was born in Cassino on 25th September 1967. He was graduated in 1991 from University of Cassino in Economics and earned his $\mathrm{Ph} . D$. in Actuarial Science from University of Rome "La Sapienza".

After teaching experiences as invited professor at the Pontifical Gregorian University (Vatican State) (1992-2000) and at University of Sassari (1997-1998), he is now full professor of Mathematics and Financial Mathematics at University of Cassino. He held the office of Head of Department from 2005 to 2009, when he resigned after the appointment as Rector's Delegate for Research and Benchmarking. He is author of about fifty research papers concerning the modeling of financial time series by (multi)fractional processes and self-similar stochastic processes.

Alexandre Pantanella was bon in Aigle (Switzerland) on 2nd December 1975. He graduated in 2003 in Mechanical Engineering from University of Cassino and earned his Ph.D. in Mathematics for Economics and Finance from University of Rome "La Sapienza" in 2009.

His research interests concern financial time series stochastic modeling and wavelet analysis.

TABLE III. RESIDUALS

\begin{tabular}{lcccccc} 
& \multicolumn{6}{c}{ Distribution parameters } \\
& Obs. & Mean & St.Dev. & Kurt & Skew & $\begin{array}{c}\text { Tail } \\
\text { index }\end{array}$ \\
\hline DJI & 1,379 & 0.0314 & 0.9952 & 2.939 & -0.2571 & -0.3349 \\
\hline FTSE & 1,384 & 0.0286 & 1.0120 & 2.748 & -0.0233 & -0.2948 \\
\hline N225 & 1,342 & 0.0073 & 1.0092 & 2.775 & -0.2172 & -0.3408 \\
\hline HSI & 1,374 & 0.0450 & 0.9984 & 2.889 & -0.1547 & -0.2913 \\
\hline
\end{tabular}

TABLE IV. JARQUE-BERA TEST FOR MPRE RESIDUALS

\begin{tabular}{|r|c|c|c|c|}
\cline { 2 - 5 } \multicolumn{1}{c|}{} & \multicolumn{4}{c|}{$\boldsymbol{p}$-values } \\
\hline Size & DJIA & FTSE & N225 & HSI \\
\hline 20 & 0.5000 & 0.5000 & 0.5000 & 0.4160 \\
\hline 40 & 0.4813 & 0.5000 & 0.2610 & 0.5000 \\
\hline 60 & 0.5000 & 0.2053 & 0.5000 & 0.1108 \\
\hline 80 & 0.5000 & 0.5000 & 0.5000 & 0.5000 \\
\hline 100 & 0.5000 & 0.5000 & 0.5000 & 0.5000 \\
\hline 120 & 0.1696 & 0.5000 & 0.5000 & 0.5000 \\
\hline 140 & 0.1321 & 0.3452 & 0.4224 & 0.4818 \\
\hline 160 & 0.1055 & 0.2685 & 0.3053 & 0.5000 \\
\hline 180 & 0.0765 & 0.1650 & 0.5000 & 0.5000 \\
\hline 200 & 0.0681 & 0.1312 & 0.5000 & 0.3973 \\
\hline 220 & 0.0513 & 0.1479 & 0.4290 & 0.2428 \\
\hline 240 & 0.0462 & 0.1506 & 0.4505 & 0.1929 \\
\hline 260 & 0.0371 & 0.1137 & 0.2606 & 0.1513 \\
\hline 280 & 0.0275 & 0.0869 & 0.2469 & 0.1462 \\
\hline 300 & 0.0237 & 0.0727 & 0.1626 & 0.1750 \\
\hline 320 & 0.0286 & 0.0665 & 0.2115 & 0.3160 \\
\hline 360 & 0.0239 & 0.0809 & 0.1561 & 0.2781 \\
\hline 400 & 0.0344 & 0.0936 & 0.1496 & 0.1646 \\
\hline 440 & 0.0382 & 0.1325 & 0.1124 & 0.1726 \\
\hline 480 & 0.0676 & 0.1068 & 0.1331 & 0.2244 \\
\hline 520 & 0.0443 & 0.2228 & 0.1804 & 0.1296 \\
\hline 560 & 0.0390 & 0.1432 & 0.1403 & 0.1126 \\
\hline 600 & 0.0310 & 0.1443 & 0.1059 & 0.1077 \\
\hline 640 & 0.0437 & 0.1782 & 0.0575 & 0.1169 \\
\hline 680 & 0.0317 & 0.1312 & 0.0442 & 0.1069 \\
\hline 720 & 0.0200 & 0.0886 & 0.0434 & 0.0662 \\
\hline 760 & 0.0181 & 0.0784 & 0.0300 & 0.1156 \\
\hline 800 & 0.0137 & 0.0610 & 0.0257 & 0.1021 \\
\hline 840 & 0.0088 & 0.0427 & 0.0207 & 0.1462 \\
\hline 880 & 0.0043 & 0.0224 & 0.0114 & 0.1388 \\
\hline 920 & 0.0040 & 0.0421 & 0.0071 & 0.1041 \\
\hline 960 & 0.0038 & 0.0471 & 0.0077 & 0.1145 \\
\hline 1,0000 & 0.0034 & 0.0219 & 0.0068 & 0.0858 \\
\hline
\end{tabular}

TABLE V. JARQUE-BERA TEST FOR GARCH(1,1) RESIDUALS

\begin{tabular}{|r|c|c|c|c|}
\cline { 2 - 5 } \multicolumn{1}{c|}{} & \multicolumn{4}{c|}{$p$-values } \\
\hline Size & DJIA & FTSE & N225 & HSI \\
\hline 20 & 0.5000 & 0.5000 & 0.5000 & 0.4395 \\
\hline 40 & 0.1907 & 0.5000 & 0.1888 & 0.1741 \\
\hline 60 & 0.2972 & 0.1220 & 0.5000 & 0.0246 \\
\hline 80 & 0.1105 & 0.2492 & 0.5000 & 0.5000 \\
\hline 100 & 0.0367 & 0.4823 & 0.5000 & 0.5000 \\
\hline 120 & 0.0070 & 0.2284 & 0.5000 & 0.5000 \\
\hline 140 & 0.0045 & 0.1182 & 0.5000 & 0.5000 \\
\hline 160 & 0.0059 & 0.0953 & 0.5000 & 0.4262 \\
\hline 180 & 0.0038 & 0.0328 & 0.5000 & 0.0210 \\
\hline
\end{tabular}


International Journal of Trade, Economics and Finance, Vol.2, No.1, February, 2011 2010-023X

\begin{tabular}{|r|c|c|c|c|}
\cline { 2 - 5 } \multicolumn{1}{c|}{} & \multicolumn{4}{c|}{$p$-values } \\
\hline Size & DJIA & FTSE & N225 & HSI \\
\hline 200 & 0.0042 & 0.0324 & 0.5000 & 0.0277 \\
\hline 220 & 0.0037 & 0.0592 & 0.5000 & 0.0239 \\
\hline 240 & 0.0027 & 0.0525 & 0.5000 & 0.0276 \\
\hline 260 & 0.0020 & 0.0509 & 0.1887 & 0.0355 \\
\hline 280 & 0.0016 & 0.0410 & 0.2006 & 0.0436 \\
\hline 300 & 0.0014 & 0.0326 & 0.1055 & 0.0590 \\
\hline 320 & 0.0019 & 0.0291 & 0.1547 & 0.1068 \\
\hline 360 & 0.0015 & 0.0233 & 0.1319 & 0.1920 \\
\hline 400 & 0.0030 & 0.0180 & 0.1396 & 0.3451 \\
\hline 440 & 0.0029 & 0.0091 & 0.0947 & 0.4419 \\
\hline 480 & 0.0041 & 0.0057 & 0.0335 & 0.3607 \\
\hline 520 & 0.0041 & 0.0085 & 0.0567 & 0.5000 \\
\hline 560 & 0.0018 & 0.0193 & 0.0739 & 0.3073 \\
\hline 600 & 0.0014 & 0.0031 & 0.0634 & 0.1933 \\
\hline 640 & 0.0020 & 0.0039 & 0.0289 & 0.1314 \\
\hline 680 & 0.0027 & 0.0010 & 0.0312 & 0.0512 \\
\hline 720 & 0.0010 & 0.0010 & 0.0356 & 0.0662 \\
\hline 760 & 0.0010 & 0.0010 & 0.0068 & 0.0718 \\
\hline 800 & 0.0010 & 0.0010 & 0.0042 & 0.0329 \\
\hline 840 & 0.0010 & 0.0010 & 0.0030 & 0.0264 \\
\hline 880 & 0.0010 & 0.0010 & 0.0010 & 0.0092 \\
\hline 920 & 0.0010 & 0.0010 & 0.0010 & 0.0107 \\
\hline 960 & 0.0010 & 0.0010 & 0.0010 & 0.0010 \\
\hline 1,0000 & 0.0010 & 0.0010 & 0.0010 & 0.0010 \\
\hline & & & & \\
\hline
\end{tabular}

